\title{
Conhecimento de cirurgiões-dentistas sobre a anatomia da face aplicada à anestesia local: uma revisão sistematizada
}

Knowledge of dentists on the anatomy of the face applied to local anesthesia: a systematic review Conocimiento de los dentistas en la anatomía de la cara aplicada a la anestesia local: una revisión sistematizada Érick Tássio Barbosa NEVES

Docente do Curso de Odontologia. Centro de Ciências, Tecnologia e Saúde. Departamento de Odontologia, Universidade Estadual da Paraíba (UEPB) 58233-000 Araruna-PB, Brasil

\section{Resumo}

Introdução: A Anatomia Humana é um componente curricular obrigatório na formação dos profissionais da saúde. Trata-se de uma disciplina básica que fornece subsídio para atividades de mais alta complexidade, como procedimentos clínicos e laboratoriais. Nesse sentido, o conhecimento da Anatomia nervosa da face se destaca como importante para a execução de uma anestesia local eficaz e segura em Odontologia. Objetivo: Avaliar a produção científica sobre o nivel de conhecimento dos cirurgiões-dentista sobre a Anatomia nervosa aplicada à anestesia local. Material e método: Trata-se de uma revisão integrativa sistematizada da literatura. Para este estudo foi realizada a busca de artigos em revistas indexadas nas bases de dados Lilacs, Scielo e Medline, utilizando como critérios de busca as seguintes palavraschave: Anestesia Local, Conhecimento, Odontologia, Neuroanatomia e Clínica Odontológica, nos idiomas português, inglês e espanhol. Resultados: Permaneceram na amostra final do estudo, 05 artigos, após avaliação da responsividade aos objetivos do estudo. A abordagem do tema é ainda incipiente na literatura e não há estudos avaliando o conhecimento anatômico de cirurgiões-dentistas sobre a anestesia local. Conclusão: Sugere-se que sejam realizados novos estudos, preferencialmente com delineamento experimental, a fim de contribuir para a educação em saúde nesta área específica e reduzir as disparidades curriculares nos centros de ensino superior.

Descritores: Anestesia Local; Conhecimento; Odontologia; Neuroanatomia; Clínicas Odontológicas.

\section{Abstract}

Introduction: Human anatomy is required in the formation process of health professionals. This is a basic course that provides knowledge for activities of higher complexity, as clinical and laboratory procedures. Anatomical Knowledge about nerves of the face stands out as important for running an effective and safe local anesthetic in Dentistry. Objective: To assess the scientific literature about the level of knowledge of dentists about nervous anatomy applied to local anesthesia. Material and method: This study is an integrative literature systematic review. For this study, scientific articles were searched in the databases Lilacs, Scielo and Medline, using as search criteria the following keywords: Local anesthesia, Dentistry, Knowledge, Neuroanatomy and Dental Clinics. Scientific articles in Portuguese, English and Spanish were included in the review. Results: Five scientific articles remained in the final sample of the study, after assessing the responsiveness to the objectives of the study. Scientific production and publication about this topic is still incipient in literature and there are no studies evaluating the anatomical knowledge of dentists on the local anesthesia. Conclusion: This study suggests that further investigation about this subject should be made, especially those with an experimental design, in order to contribute to health education in this specific area and reduce the disparities in higher education.

Descriptors: Anesthesia, Local; Knowledge; Dentistry; Neuroanatomy; Dental Clinics.

\section{Resumen}

Introducción: La anatomía humana es un componente curricular necesario en la formación de profesionales de la salud. Se trata de una disciplina básica que proporciona la asignación para actividades de mayor complejidad, como clínicas y procedimientos de laboratorio. En este sentido, el conocimiento de la anatomía del rostro nervioso se destaca como importante para la aplicación de un anestésico local eficaz y seguro en la odontología. Objetivo: evaluar la literatura científica sobre el nivel de conocimiento del odontólogo sobre la anatomía del nervioso aplicada a la anestesia local. Material y métodos: una revisión sistemática de la literatura Integrativa. Para este estudio se llevó a cabo para buscar artículos en revistas indexadas en las bases de datos Lilacs y Scielo, Medline, utilizando como criterio de búsqueda las siguientes palabras clave: anestesia Local, odontología, conocimiento y neuroanatomía clínica Dental, Portugués, Inglés y español. Resultados: Seguía habiendo en la muestra final del estudio, 05 artículos, después de evaluar la capacidad de respuesta a los objetivos del estudio. El enfoque del tema es aún incipiente en la literatura y no hay estudios que evalúen el conocimiento anatómico de los dentistas en el local. Conclusión: se sugiere hacer estudios adicionales preferentemente con diseño experimental, con el fin de contribuir a la educación para la salud en esta área específica y reducir las disparidades en el currículo de la educación superior.

Descriptores: Anestesia Local; Conocimiento; Odontología; Neuroanatomía; Clínicas Odontológicas.

INTRODUÇÃO

A Anatomia Humana é um componente curricular obrigatório na formação dos profissionais da saúde e permite um dos primeiros contatos dos ingressantes com a profissão escolhida. $\mathrm{O}$ reconhecimento de estruturas normais do corpo humano implica em aplicações práticas e auxilia o exercício profissional ${ }^{1,2}$.

A formação no curso de Odontologia inclui o estudo detalhado da Anatomia da cabeça e do pescoço que é importante para o diagnóstico de alterações orofaciais, auxilia a tomada de decisões clínicas e a interpretação de exames radiográficos ${ }^{3}$.

$\mathrm{O}$ estudo da Anatomia do sistema estomatognático é essencial para a realização de diversos procedimentos odontológicos. Dentres esses procedimentos encontra-se a anestesia local que é a perda temporária da condução nervosa impedindo a ocorrência da dor e dessa forma favorecendo a execução de procedimentos de maneira confortável. Para que seja realizada uma anestesia local apropriada, é importante que o profissional seja capaz de reconhecer variações anatômicas e detalhes da anatomia óssea, nervosa, vascular e de superfície da região, potencializando assim as chances de sucesso $^{4,5}$.

Apesar dessa alta relevância, pouco tem-se atentado para a avaliação do conhecimento obtido por cirurgiões-dentistas sobre anatomia nervosa direcionado para a anestesia local. Por tratar-se de uma prática comum e essencial à profissão, essa avaliação poderia ser útil para redirecionar as estratégias educacionais ou validar as metodologias já aplicadas nos centros de ensino superior.

Mais de dois terços dos cirurgiões-dentistas 
já realizaram atendimentos de parestesias e outras injúrias nervosas após o atendimento odontológico, especialmente após exodontias dos terceiros molares inferiores. Há também relatos na literatura de variações anatômicas de nervos ou estruturas relacionadas, responsáveis por erros profissionais. Dessa forma, é essencial que o cirurgião-dentista detenha conhecimentos anatômicos diversos para a prática clínica segura, estendendo-se essa concepção a todas as especialidades odontológicas ${ }^{6-8}$.

Portanto, o objetivo desse estudo foi realizar uma revisão integrativa da literatura com a finalidade de avaliar se há na literatura estudos que avaliem o conhecimento dos cirurgiões-dentistas acerca da Anatomia nervosa da cabeça e do pescoço com ênfase na anestesia local e se esse conhecimento tem sido satisfatório para a prática da profissão.

\section{MATERIAL E MÉTODO}

Trata-se de uma revisão integrativa da literatura realizada de forma sistematizada em seis fases: elaboração da pergunta norteadora, busca na literatura, coleta de dados, análise crítica dos artigos incluídos, discussão dos resultados e apresentação da revisão integrativa ${ }^{9}$.

A questão norteadora desse estudo foi se há informações na literatura sobre a importância do conhecimento sobre a Anatomia nervosa da face para a prática clínica da anestesia local em Odontologia. Foi realizada uma busca de artigos científicos indexados nas bases de dados da Literatura LatinoAmericano e do Caribe em Ciências da Saúde (LILACS), Scientific Eletronic Library Online (SCIELO) e da Medical Literature Analysis and Retrieval System Online (MEDLINE). Foram utilizados os seguintes Descritores em Ciências da Saúde (DECS) em inglês e português: Anesthesia, Local (Anestesia Local), Conhecimento (Knowledge), Odontologia (Dentistry), Neuroanatomia (Neuroanatomy) e Clínica Odontológica (Dental Clinics).

A pesquisa de artigos foi realizada independentemente do ano de publicação e desenho do estudo. Os critérios de inclusão foram artigos publicados em português brasileiro, inglês ou espanhol, estudos abordando de maneira direta ou indireta o tema Anatomia nervosa para cirurgiões dentistas com aplicação para anestesia local. Os critérios de exclusão foram artigos não relacionados ao tema norteador do estudo e artigos em outros idiomas que não os mencionados. Foi realizada inicialmente a leitura do título e resumo dos artigos, aqueles que responderam aos objetivos do estudo foram então lidos na íntegra para compor a amostra deste trabalho. Os dados foram posteriormente tabulados, conforme as seguintes informações: autoria e ano do estudo, desenho do estudo, objetivo, resultados e implicações do estudo.

\section{RESULTADOS}

A busca de artigos resultou em 15 estudos. Após a aplicação dos critérios de elegibilidade, 05 artigos foram incluídos nesta revisão integrativa sistematizada, compondo a amostra final. Destes, 04 foram encontrados na base de dados Lilacs e 01 na base de dados Scielo. Com relação ao idioma em que os trabalhos foram escritos, resultou-se em 02 artigos em inglês, 02 artigos em português e 01 artigo em espanhol (Figura 1).

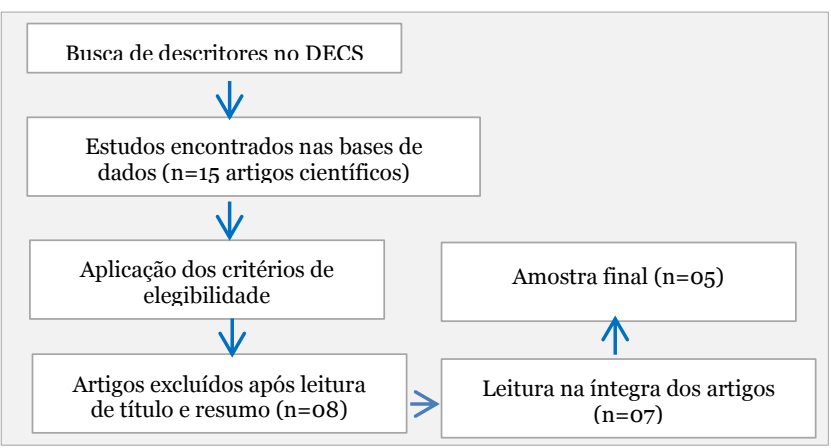

Figura 1: Fluxograma com o processo de seleção dos artigos para compor a revisão integrativa sistematizada

Os artigos que compuseram a amostra final do estudo foram tabulados e organizados de acordo com informações importantes para investigação da questão norteadora (Tabela 1).

Tabela 1 - Descrição dos artigos incluídos na revisão integrativa da literatura conforme responsividade à questão norteadora inicial do estudo

\begin{tabular}{|c|c|c|c|c|}
\hline $\begin{array}{c}\text { Autor/ } \\
\text { Ano }\end{array}$ & $\begin{array}{l}\text { Desenho do } \\
\text { Estudo }\end{array}$ & Objetivo & Resultados & Implicações \\
\hline $\begin{array}{l}\text { Bolaños et al. }{ }^{10} \\
\quad 2015\end{array}$ & $\begin{array}{l}\text { Revisão de } \\
\text { literatura }\end{array}$ & $\begin{array}{l}\text { Discutir causas } \\
\text { associadas ao } \\
\text { fracasso do } \\
\text { bloqueio } \\
\text { anestésico local }\end{array}$ & $\begin{array}{c}\text { Variações } \\
\text { anatômicas } \\
\text { (nervos bífidos ou } \\
\text { forames } \\
\text { acessórios) } \\
\text { podem ser } \\
\text { encontradas }\end{array}$ & $\begin{array}{l}\text { Dentre outros } \\
\text { fatores, as variações } \\
\text { anatômicas podem } \\
\text { dificultar o sucesso } \\
\text { da anestesia local }\end{array}$ \\
\hline $\begin{array}{c}\text { Vasconcelos et al. } .^{11} \\
2007\end{array}$ & $\begin{array}{l}\text { Revisão de } \\
\text { literatura }\end{array}$ & $\begin{array}{l}\text { Demonstrar o } \\
\text { risco de } \\
\text { aspirações } \\
\text { positivas } \\
\text { decorrente do } \\
\text { bloqueio do } \\
\text { nervo alveolar } \\
\text { inferior }\end{array}$ & $\begin{array}{c}\text { Aspiração antes da } \\
\text { anestesia previne a } \\
\text { injeção } \\
\text { intravascular e } \\
\text { ocorrência de } \\
\text { reações adversas } \\
\text { atribuídas à } \\
\text { overdose }\end{array}$ & $\begin{array}{c}\text { Conhecimentos da } \\
\text { anatomia vascular, } \\
\text { nervosa e da técnica } \\
\text { de aspiração do } \\
\text { anestésico reduzem } \\
\text { risco de toxicidade } \\
\text { durante anestesia } \\
\text { local }\end{array}$ \\
\hline $\begin{array}{l}\text { Mattos et al. }{ }^{12} \\
\quad 1999\end{array}$ & $\begin{array}{l}\text { Revisão de } \\
\text { literatura }\end{array}$ & $\begin{array}{l}\text { Discutir } \\
\text { complicaçôes da } \\
\text { anestesia local } \\
\text { em crianças }\end{array}$ & $\begin{array}{l}\text { Complicações da } \\
\text { anestesia local são } \\
\text { frequentes e } \\
\text { muitas vezes com } \\
\text { sintomas brandos }\end{array}$ & $\begin{array}{l}\text { O cirurgião-dentista } \\
\text { necessita de } \\
\text { conhecimento da } \\
\text { literatura e bom } \\
\text { senso para realizar } \\
\text { anestesia local } \\
\text { segura e eficaz }\end{array}$ \\
\hline Palti et al..$^{13} 2011$ & $\begin{array}{l}\text { Delineamento } \\
\text { experimental }\end{array}$ & $\begin{array}{c}\text { Descrever } \\
\text { técnica } \\
\text { alternativa para } \\
\text { bloqueio do } \\
\text { nervo alveolar } \\
\text { inferior } \\
\text { utilizando } \\
\text { diversos pontos } \\
\text { anatomicos de } \\
\text { referência }\end{array}$ & $\begin{array}{l}\text { A proposta de uma } \\
\text { técnica alternativa } \\
\text { para localização do } \\
\text { nervo alveolar } \\
\text { inferior } \\
\text { demonstrou-se um } \\
\text { método efetivo } \\
\text { para anestesia local }\end{array}$ & $\begin{array}{l}\text { Uma anestesia } \\
\text { adequada do nervo } \\
\text { alveolar inferior } \\
\text { depende de } \\
\text { conhecimentos } \\
\text { prévios e referências } \\
\text { em pontos } \\
\text { anatômicos }\end{array}$ \\
\hline $\begin{array}{l}\text { Blanton et al. } .^{14} \\
2003\end{array}$ & $\begin{array}{l}\text { Revisão de } \\
\text { literatura }\end{array}$ & $\begin{array}{c}\text { Discutir sobre a } \\
\text { distribuicicão } \\
\text { periférica do } \\
\text { nervo trigêmio } \\
\text { para estimular } \\
\text { uma anestesia } \\
\text { local segura e } \\
\text { efetiva }\end{array}$ & $\begin{array}{c}\text { A anestesia } \\
\text { apropriada do } \\
\text { nervo trigêmio é } \\
\text { um pre requisito } \\
\text { para uma } \\
\text { adequada e } \\
\text { duradoura } \\
\text { anestesia local }\end{array}$ & $\begin{array}{c}\text { O cirurgião-dentista } \\
\text { deve conhecer a } \\
\text { anatomia nervosa, } \\
\text { vascular e óssea e } \\
\text { reconhecer } \\
\text { potenciais } \\
\text { implicaçóses de } \\
\text { variçóes } \\
\text { anatômicas }\end{array}$ \\
\hline
\end{tabular}

DISCUSSÃO

Os estudos selecionados para esta revisão integrativa sistematizada da literatura após os critérios de inclusão foram principalmente artigos de revisão de literatura, com exceção de um estudo de desenho experimental. De forma geral, os autores 
estudados afirmam que o controle da dor possui papel de destaque na Odontologia e é essencial para o sucesso de diversos procedimentos clínicos, principalmente os procedimentos de natureza cirúrgica. Nesse sentido, condutas que objetivam redução ou suspensão da dor na região orofacial demandam conhecimento da neuroanatomia, especialmente das estruturas nervosas que se localizam na cabeça e no pescoço ${ }^{14,15}$.

Um estudo prévio, realizado com graduandos em Odontologia, avaliou o conhecimento dos alunos sobre a utilização de anestésicos locais em pacientes com necessidades especiais. $\mathrm{O}$ estudo realizou questões sobre a seleção apropriada de substâncias anestésicas de acordo com diferentes grupos de comorbidades. Concluiu-se que o conhecimento dos alunos foi deficiente e limitado. Não houve nesse estudo, perguntas relacionadas a critérios anatômicos durante a anestesia local, uma vez que o objetivo principal foi direcionado a critérios farmacológicos. De maneira geral, outros estudos também tem demonstrado que existem dificuldades dos profissionais em termos de conhecimento científico quanto à técnica anestésica, o que pode sugerir negligência por parte dos envolvidos ou falha no processo educativo $^{16,17}$.

Com base nos estudos incluídos na presente revisão da literatura, pode-se perceber que a observação da anatomia da região é um pressuposto necessário para prática da anestesia em Odontologia, pois a sua não observação leva a complicações e acidentes que prejudicam uma boa condução dos tratamentos. Em concordância com essa ideia, estudos tem sugerido que estão entre as mais frequentes razões de falhas anestésicas: a falta de observação do posicionamento das estruturas anatômicas como nervos, forames e vasos sanguíneos ${ }^{7,10}$.

Não foram observados estudos avaliando o nível de conhecimentos de dentistas sobre a anatomia da face $e$ as possíveis implicações desse conhecimento na prática anestésica. Esta lacuna na literatura assume uma importante função, pois apesar da crescente prevalência de falhas no processo de anestesia local, não tem-se destinado atenção especial para as prováveis causas anatômicas desse processo $^{12,13}$.

Acredita-se que uma melhoria na capacidade técnica dos profissionais pode estar relacionada ao processo educativo nos centros de ensino. Percebe-se uma crescente tentativa de modernização do processo de ensino-aprendizagem, incorporando novas tecnologias de ensino e processos didáticoconstrutivos. Embora um estudo prévio tenha avaliado a utilização de uma estratégia específica na melhoria do ensino da Anatomia (mapas conceituais) e obtido resultados satisfatórios ${ }^{2,18}$, não é do nosso conhecimento a presença de outros estudos avaliando os resultados de métodos de ensino em áreas específicas como na Anatomia aplicada à anestesia local em Odontologia. Estes estudos são importantes pois podem permitir a formulação de estratégias educativas ou reprodução de metodologias potencialmente vantajosas, melhorando os resultados e desempenhos clínicos dos cirurgiões-dentistas.

\section{CONCLUSÃO}

A partir dos estudos incluídos nessa revisão integrativa da literatura percebeu-se que há uma abordagem moderada acerca da importância da Anatomia para a aplicação adequada de uma anestesia local em Odontologia, porém não foram encontrados estudos sobre o nível de conhecimento dos cirurgiões-dentistas acerca do tema, o que indica uma lacuna científica a ser melhor explorada em estudos futuros, especialmente com desenhos experimentais.

\section{REFERÊNCIAS}

1. Arruda RM, Sousa CRA. Aproveitamento teóricoprático da disciplina Anatomia Humana do curso de Fisioterapia. Rev Bras Educ Médica. 2014; 38(1):65-71.

2. Mouthé Filho A, Borges MAS, Figueiredo IPR, Villalobos MIOB, Taitison PF. Refletindo o ensino da Anatomia Humana. Enfermagem Revista. 2016; 19(2):169-175.

3. Antoniazzi MCC, Carvalho PL, Koide CH. Importância do conhecimento da anatomia radiográfica para a interpretação de patologias ósseas. Revista Gaúcha de Odontologia. 2008; 56(2):195-199.

4. Silva SREP, Andrade APRCB, Costa FP, Cunha RS, Politano GT, Pinheiro SL, Imparato JCP. Avaliação da técnica anestésica local utilizada por alunos de graduação em Odontologia. Con Scientiae Saúde. 2010; 9(3):469-475.

5. Teixeira LMS, Reher P, Reher VGS. Anatomia aplicada à Odontologia. Rio de Janeiro. 2ed. Guanabara Koogan, 2008.

6. Lopes GB, Freitas JB. Parestesia do nervos alveolar inferior após exodontia de terceiros molares. Arquivos Brasileiro de Odontologia. 2013; 9(2):35-40.

7. Andrade YDN, Araujo EBJ, Souza LMA, Groppo FC. Análise das variações anatômicas do canal da mandíbula encontradas em radiografias panorâmicas. Revista Odontol Unesp. 2015; 44(1)31-36.

8. Dodo CG, Sotto-Maior BS, Faot F, Del Bel Cury AA, Senna PM. Lesão do nervo alveolar inferior por implantes dentários: prevenção, diagnóstico e tratamento. Dental impress Implantol. 2015; 9(4)57-66.

9. Souza MT, Silva MD, Carvalho R. Revisão integrativa: o que é e como fazer. Einstein. 2010; 8(1):102-106. 
10.Bolanos DC, Wong LR, Guillén AJP. Comprendiendo y combatiendo el fracasso anestésico en Odontología. Revista ADM. 2015; 72(6):290-98.

11. Vasconcelos BCE, Freitas KCM, Almeida RAC, Mauricio HA. A importância da técnica de aspiração prévia ao bloqueio anestésio do nervo alveolar inferior. Rev Cir Traumatol BucoMaxilo-Fac. 2007;7(1):29-36.

12. Mattos ABT, Gleiser R, Primo LSSG. Complicações anestésicas em Odontopediatria. J bras odontopediatr odontol bebê. 1999; 2(5):49-56.

13.Palti DG, Almeida CM, Rodrigues AC, Andreo JC, Lima JEO. Anesthetic technique for inferior alveolar nerve block: a new approach. J Appl Oral Sci. 2011;19(1):11-15.

14.Blanton PL, Jeske AH. The key to profound local anesthesia: neuroanatomy. J Am Dent Assoc. 2003; 134(6):753-60.

15.Cabral ED. Dental local anesthesia in family health units: use, pain and associated factors. Rev Dor. 2015; 16(4):254-58.

16.Pontanegra RSM, Camboim CCL, Freire JCP, Nóbrega MCT, Barreto JO, Santos JA, DiasRibeiro E. Análise do conhecimento de graduandos em Odontologia sobre o uso de anestésico local em pacientes com necessidades especiais. Faculdade de Odontologia de Lins/Unimep. 2017; 27(1):5-14.

17. Carvalho B, Fritzen EL, Parodes AG, Santos RB, Gedoz L. O emprego dos anestésicos locais em Odontologia: Revisão de Literatura. Rev bras odontol. 2013;70(2):178-81.

18. Foreaux G, Sá MA, Schetino LPL, Guerra LB, Silva JH. O ensino-aprendizagem da anatomia humana: avaliação do desempenho dos alunos após a utilização de mapas conceituais como uma estratégia pedagógica. Ciênc Educ. 2018; 24(1):95-110.

\section{CONFLITO DE INTERESSES}

Os autores declaram não haver conflitos de interesse.

AUTOR PARA CORRESPONDENCIA

Erick Tássio Barbosa Neves

erick.tassio@hotmail.com

Submetido em 09/07/2018

Aceito em 04/10/2018 